\title{
Analysis of Fabry-Perot Velocimeter Records
}

\author{
G. Avara
}

August 3, 2001

U.S. Department of Energy

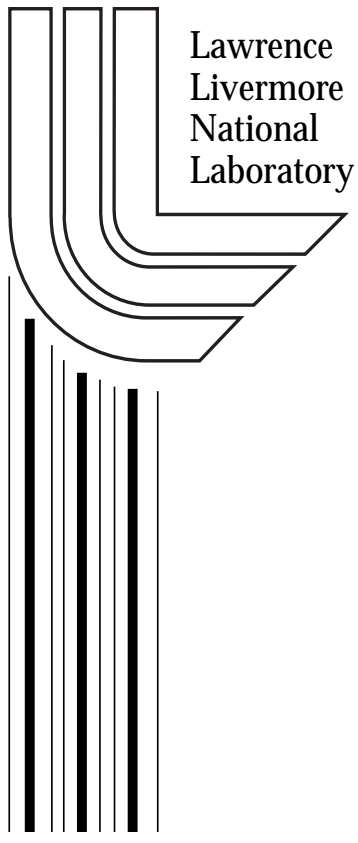




\section{DISCLAIMER}

This document was prepared as an account of work sponsored by an agency of the United States Government. Neither the United States Government nor the University of California nor any of their employees, makes any warranty, express or implied, or assumes any legal liability or responsibility for the accuracy, completeness, or usefulness of any information, apparatus, product, or process disclosed, or represents that its use would not infringe privately owned rights. Reference herein to any specific commercial product, process, or service by trade name, trademark, manufacturer, or otherwise, does not necessarily constitute or imply its endorsement, recommendation, or favoring by the United States Government or the University of California. The views and opinions of authors expressed herein do not necessarily state or reflect those of the United States Government or the University of California, and shall not be used for advertising or product endorsement purposes.

This work was performed under the auspices of the U. S. Department of Energy by the University of California, Lawrence Livermore National Laboratory under Contract No. W-7405-Eng-48.

This report has been reproduced directly from the best available copy.

Available electronically at http://www.doc.gov/bridge

Available for a processing fee to U.S. Department of Energy

And its contractors in paper from

U.S. Department of Energy

Office of Scientific and Technical Information

P.O. Box 62

Oak Ridge, TN 37831-0062

Telephone: (865) 576-8401

Facsimile: (865) 576-5728

E-mail: reports@adonis.osti.gov

Available for the sale to the public from

U.S. Department of Commerce

National Technical Information Service

5285 Port Royal Road

Springfield, VA 22161

Telephone: (800) 553-6847

Facsimile: (703) 605-6900

E-mail: orders@ntis.fedworld.gov

Online ordering: http://www.ntis.gov/ordering.htm

OR

Lawrence Livermore National Laboratory

Technical Information Department's Digital Library

http://www.llnl.gov/tid/Library.html 


\title{
Analysis of Fabry-Perot Velocimeter Records
}

\begin{abstract}
Program demonstration and user instructions are presented for FabryVB5. This computer program was created for use in analyzing Fabry-Perot interferometer records that detail the velocity time histories of fast moving surfaces. Graphical curves representing peak fringe positions and fiducial timing dots are extracted from a digitized film record or from a CCD digital image. An analysis is demonstrated on a sample velocimeter record along with some mathematical formula and routine operations. Routines used to analyze calibration records on streak camera distortions are illustrated in an appendix. This is a Microsoft Visual Basic ${ }^{\mathrm{TM}}$ version for the PC.
\end{abstract}

\section{Introduction}

To determine velocities of fast moving objects, film records of Doppler shifted interference fringes are obtained from the surface reflected laser light. ${ }^{1}$ Experiments performed at the Lawrence Livermore National Laboratory (LLNL) utilize a Many Beam Fabry Perot Velocimeter to acquire velocities from various positions on a given surface. ${ }^{2}$ Several of these five beam velocimeters are used at LLNL diagnostic facilities at Livermore and Nevada. Laser beams that reflect from five separate surface positions are multiplexed through a single interferometer, and the associated optics (see Fig. 1), to form five interference fringes which are recorded on separate electronic streak cameras. A single recorded image is shown in Fig. 2 where the camera sweeps in time along the horizontal direction from left to right. Interference fringes are measured to calculate the velocity history.

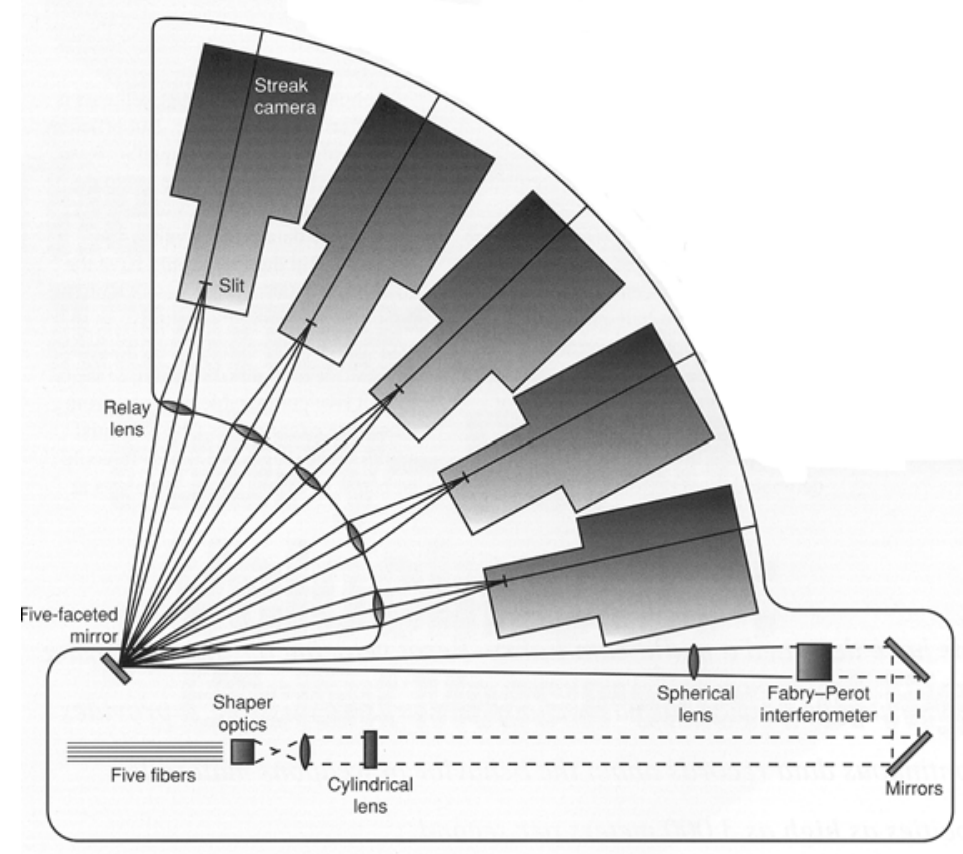

Figure 1. Many Beam Velocimeter 


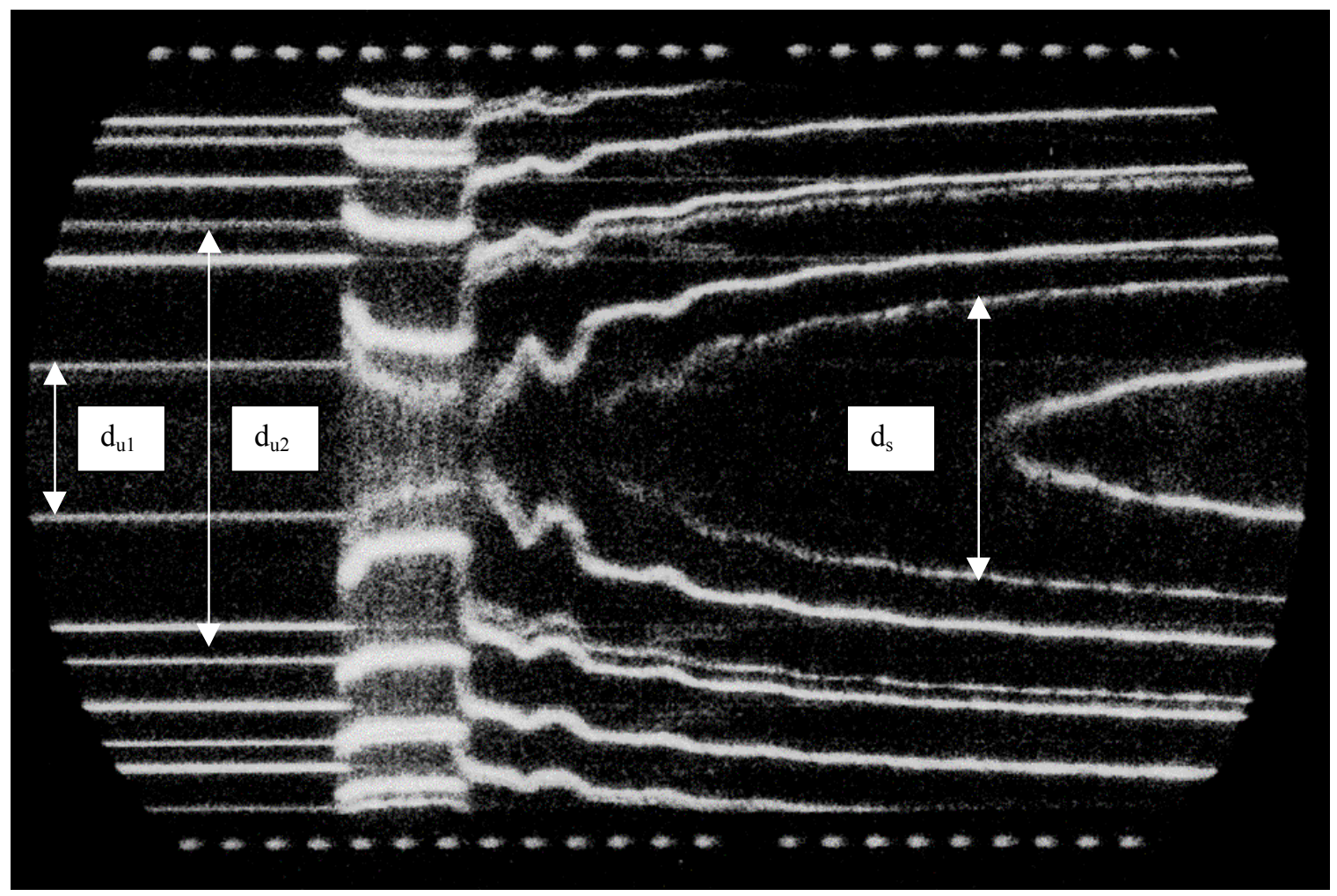

Figure 2. Sample (Double Cavity) Velocity Fringe Record

The straight-line fringes on the left are called UN-Doppler shifted lines (light) while the curvy lines on the right are called Doppler shifted lines. The program operates on both sets of these lines to calculate velocity using the following formula.

$$
V=(c \lambda / 4 h)(i+\delta) .
$$

Where $\delta=\left(\mathrm{d}_{\mathrm{s}}{ }^{2}-\mathrm{d}_{\mathrm{u} 1}{ }^{2}\right) /\left(\mathrm{d}_{\mathrm{u} 2}{ }^{2}-\mathrm{d}_{\mathrm{u} 1}{ }^{2}\right)$.

The subscript using $s$ represents a Doppler shifted line and the subscripts using $u$ are UN-Doppler shifted lines. The $d$ symbols are diameters measured vertical across fringes where subscripts 1 and 2 are respective inner and outer adjacent fringe sets. Laser light traveling with velocity $c$ has the wavelength $\lambda$. The interference fringe pattern jumps through $i$ fringe formations before the velocity change is small enough for new visible fringes to be recorded. The value $(\mathrm{c} \lambda / 4 \mathrm{~h})$ is usually called the fringe constant where $h$ is the cavity length of the Fabry-Perot interferometer.

Equation (1) is the only formula used by the program to calculate velocities. The program actually uses the radius $r$ (rather than diameter $d$ ) so that each individual curve can be accounted for. A radius is measured at the peak optical density (maximum light exposure) from a given fringe. Other program routines (discussed in Appendix A) use various mathematical corrections to minimize effects of streak camera distortions. These distortions and their effects on velocity and time measurements are presented in a previous paper. ${ }^{3}$

An analysis on the sample of Fig. 2 will demonstrate the various routines available to the user of this program. For clarity, some of the program's mathematical equations will be given. However, most routines will be described conceptually for their functions. This paper is intended to serve as a user manual. 


\section{Reading Bitmap Images}

We begin an analysis by reading up the sample bitmap image from the File / Open menu (see Fig. 3). Filters on the file types can be used to view only bitmap files. Select Fids from the Digitize menu (see Fig. 4). Now click (and release) on the upper left, then lower right regions of the fids. A rectangle will be drawn around the selected region and curve fit routines will fit to the $X$ and $Y$ cross sections to find the peak optical density for each fid dot. A poor reading can be removed by selecting Remove Last Reading from the Digitize menu. The Digitize menu pops up with a right mouse click or by using normal menu selection.

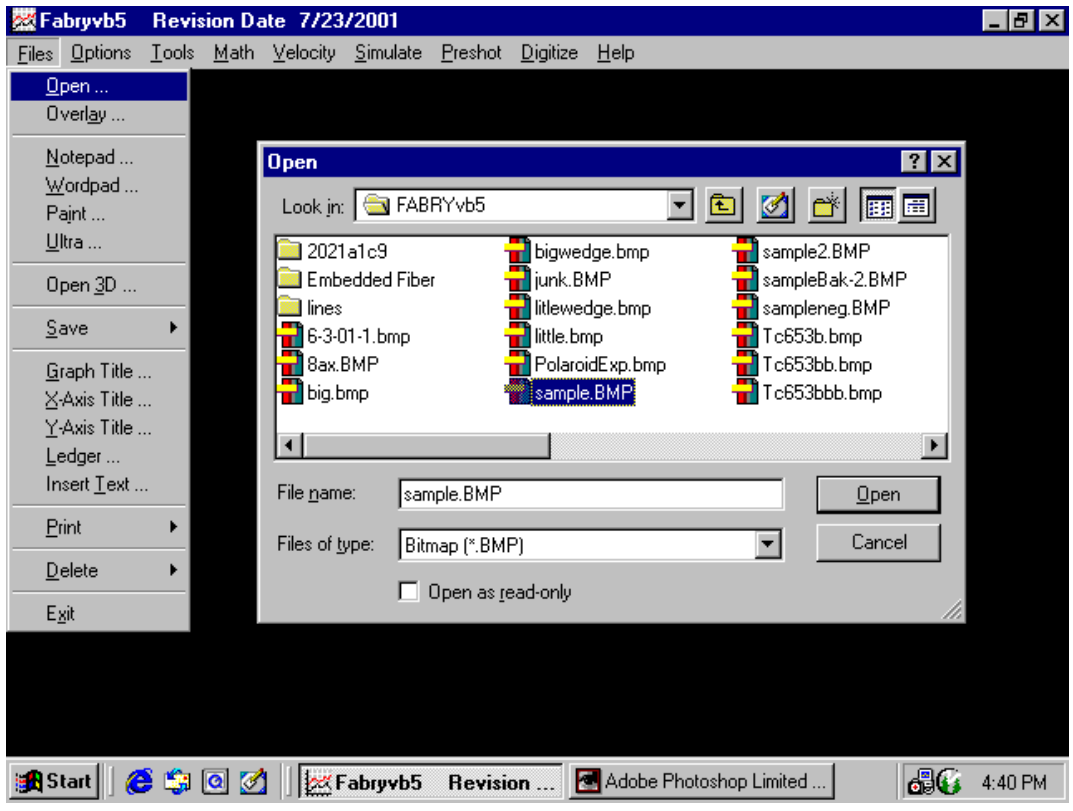

Figure 3. Opening a Bitmap File

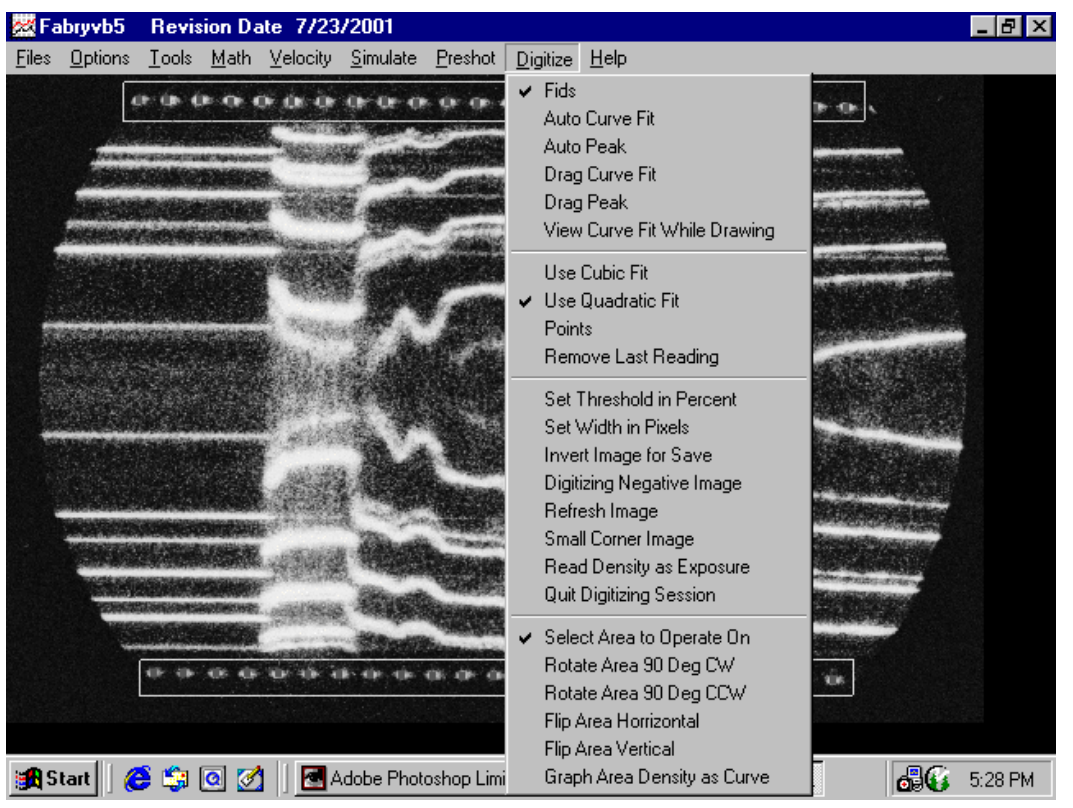

Figure 4. Reading the Fids 
Adjustments on Threshold and Width can be made from the Digitize menu to optimize readings. Default threshold is set at $2 \%$ (of maximum density) and default width is set at 10 pixels. Set the width to match the fid width or fringe broadness that is being read.

Once the top and bottom fids are read, we read the UN-Doppler fringes. Select Auto Curve Fit for easy to read fringes. The Auto $P$ eak function is more robust for weaker fringes. These two functions operate from mouse clicks on the beginning and end points of a fringe. Both Curve Fit functions mathematically fit a curve to the fringe's cross section profile of optical density. This fit can be seen by selecting View Curve Fit While Drawing (see Fig. 5) from the Digitize menu. Do not use this function during a reading that will be saved. This function allows the user to view a curve fit for proper selection of the Width and Threshold settings.

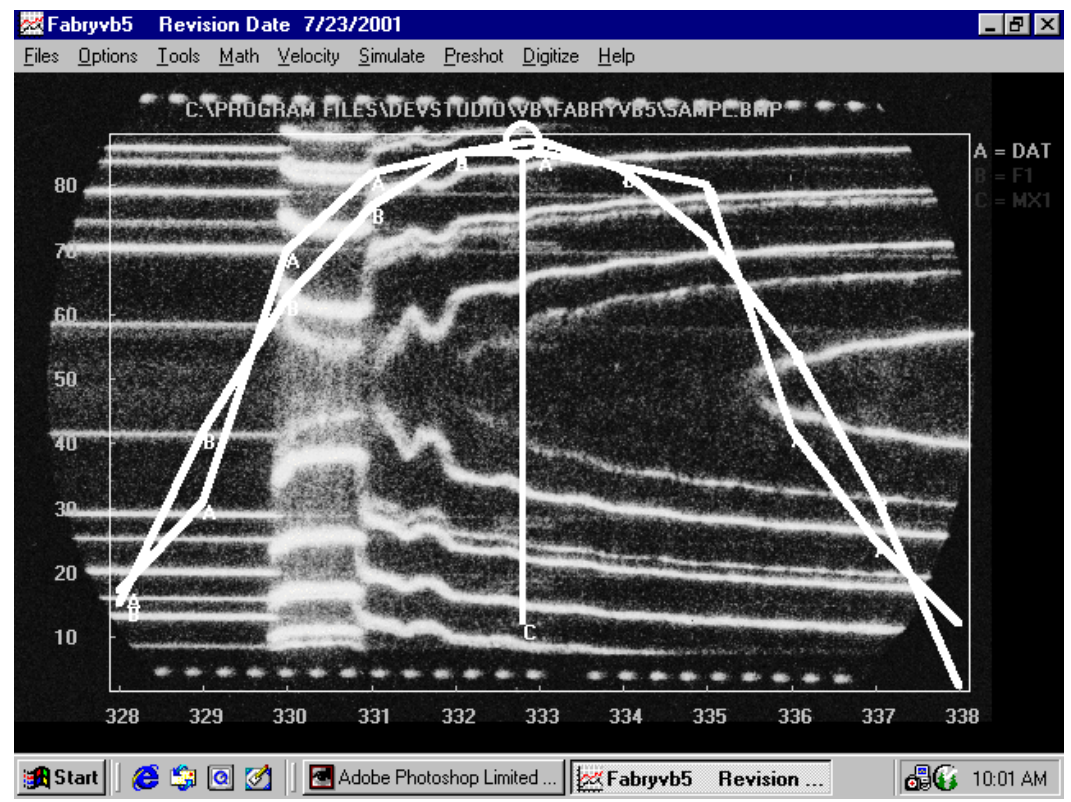

Figure 5. View Curve Fit While Drawing

The most difficult fringes can be read using the Drag functions. Drag Curve Fit produces a smoother read but the Drag Peak can read weak and broken fringes. Both Drag functions work well when there are neighboring fringes that are very close to each other. The Points function can be used to select individual points to be saved as a curve. Points are recorded exactly at each mouse click and are not curve fit to a peak. The same functions just described are also used to read Doppler shifted lines. Lines or fids can be read in any order. The user can choose either Quadratic or Cubic curve fit by selection from the Digitize menu. Images can also be saved as negatives and then read back up for analysis. When reading a negative image, the user must select Digitizing Negative Image from the menu.
Selecting Refresh reads the current clean image back up without clearing out any data that has been acquired.

Choosing Read Density as Exposure converts all pixel depth values from density to exposure while a reading is in process. This has the effect of sharpening peak regions relative to low exposure regions. Its usefulness can only be found by trial and error. An optical density step wedge curve (named wedge.ult) must be provided and can be read from film records using the Select Area to Operate On function followed by selection of the Graph Area as Density function. Flip and Rotation functions are provided for simple orientation of images. Once all fringes are read, select Quit Digitizing Session from the menu. 


\section{Analyzing Records}

Selecting Quit Digitizing Session quits digitizing and reads up a binary file with the .ult extension (see Fig. 6). The fringe file in the background was just read from the bitmap image. Selecting Small Corner Image from the Digitize menu can bring up a small bitmap image in the upper left corner. Selecting again removes the small image.

We begin the analysis by selecting some user options from the Velocity Analysis Options menu. Fig. 6 shows some choices selected for a typical analysis. The first block of options allows user control on cavity sorting, velocity jump selection and double record analysis. Selecting Single Record Auto Analysis starts the actual analysis.

Selecting the option Interrupt After Cavity Sort allows the user to inspect program results on interferometer cavity sorting before allowing the program to proceed through the remaining analysis. If something is wrong, the user can fix the problem and then proceed by re-selecting Single Record Analysis. Selecting STOP Auto Analysis aborts the analysis.
The program typically determines velocity jump conditions automatically from two interferometer cavity lengths. Without a double cavity interferometer, the user must know the actual velocity at surface jump off within half of the fringe constant. When a double cavity is used, then the user must know the true jump velocity within a span of several fringe constants. By overlaying several possible solutions and choosing a velocity with common solutions from the two cavity lengths, the program can usually choose the correct velocity curve.

The second block of options controls the use of distortion correction curves. Selecting Skip Un-Distort causes the program to skip all distortion corrections, including the use of top and bottom fids for corrections. The third options block selects various routines that the program performs to determine the quality of a completed analysis. Choosing the options shown in Fig. 6 is a good first choice for performing an analysis.

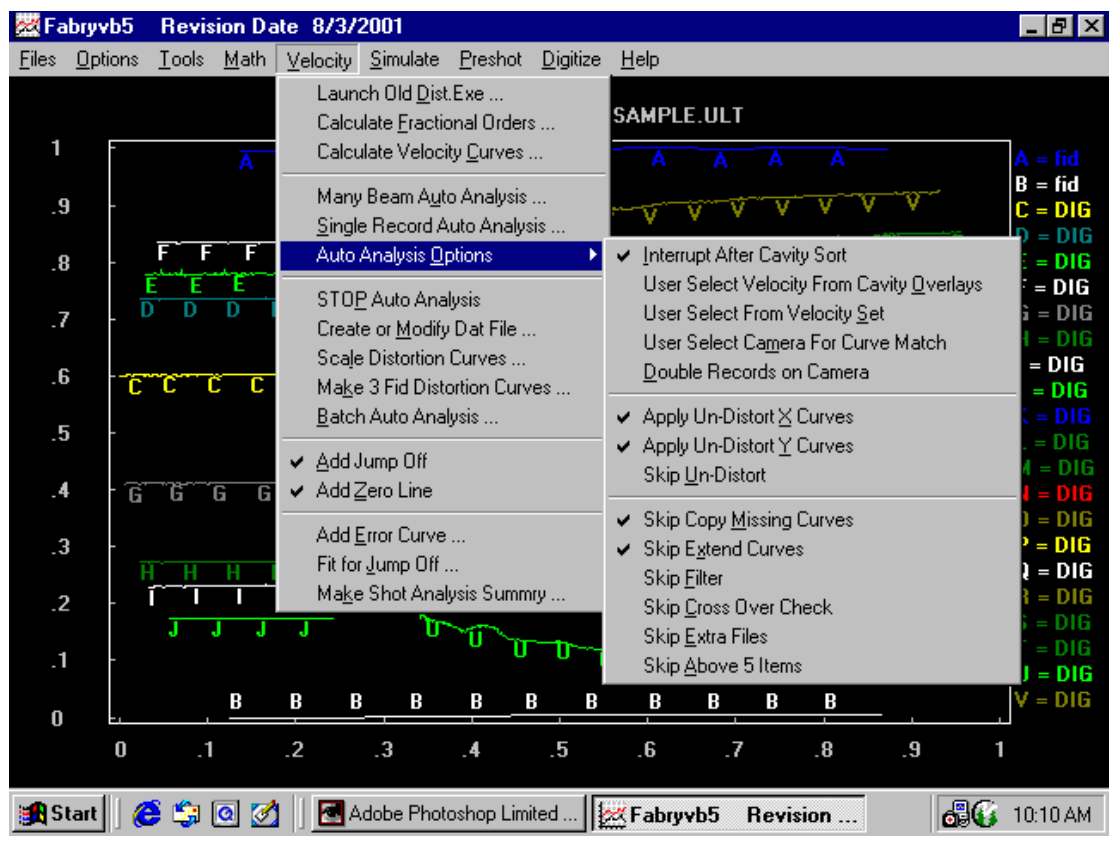

Figure 6. Velocity Analysis Options 
Selecting Single Record Auto Analysis brings up the sample.dat file (see Fig. 7) to be edited for the auto analysis routines.

After scaling the data and performing distortion corrections, the program separates the double cavity record into two separate files named sample.ca and sample.cb.

A set of velocity curves (sample.cav and sample.cbv) is calculated from each file and the two sets are overlaid (see Fig. 8). A set of four nearest matching velocity curves is selected from this overlay and saved in the file sample.mat. If the previously mentioned options are selected, a single nearest match velocity curve is saved as sample.udv.

Should the user choose the User Select Velocity From Cavity Overlay option, then the program would allow the user to select from the overlay of sample.cav and sample.cbv. The User Select From Velocity Set option allows the user to choose from the four curves in the file sample.mat. The User Select Camera For Curve Match option lets the user overlay a previously determined correct velocity curve for matching. In this case, the current record to be analyzed must be a single cavity record.

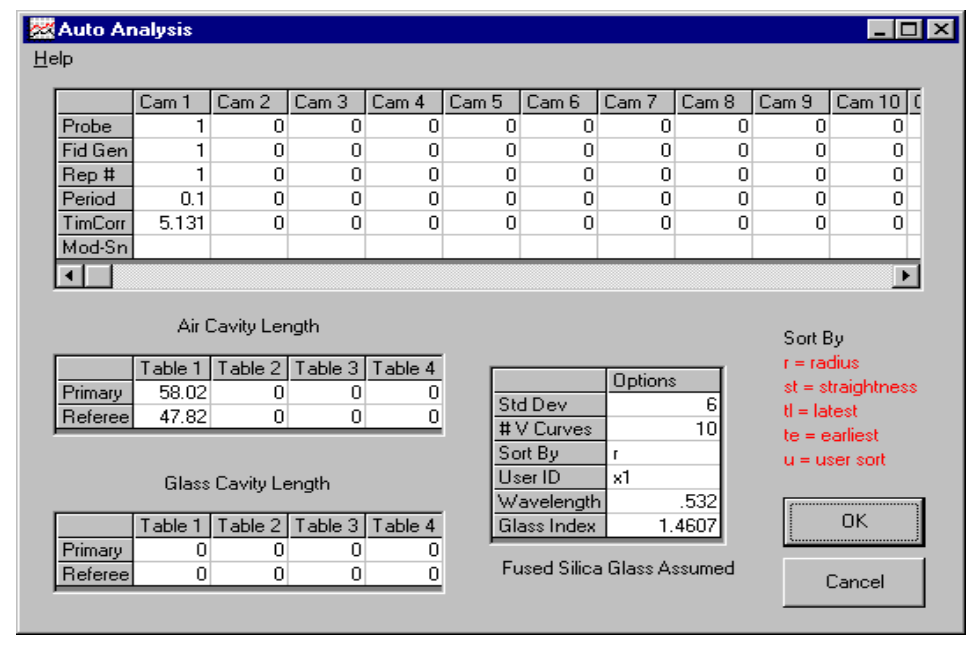

Figure 7. Editing sample.dat for the Auto Analysis Routines

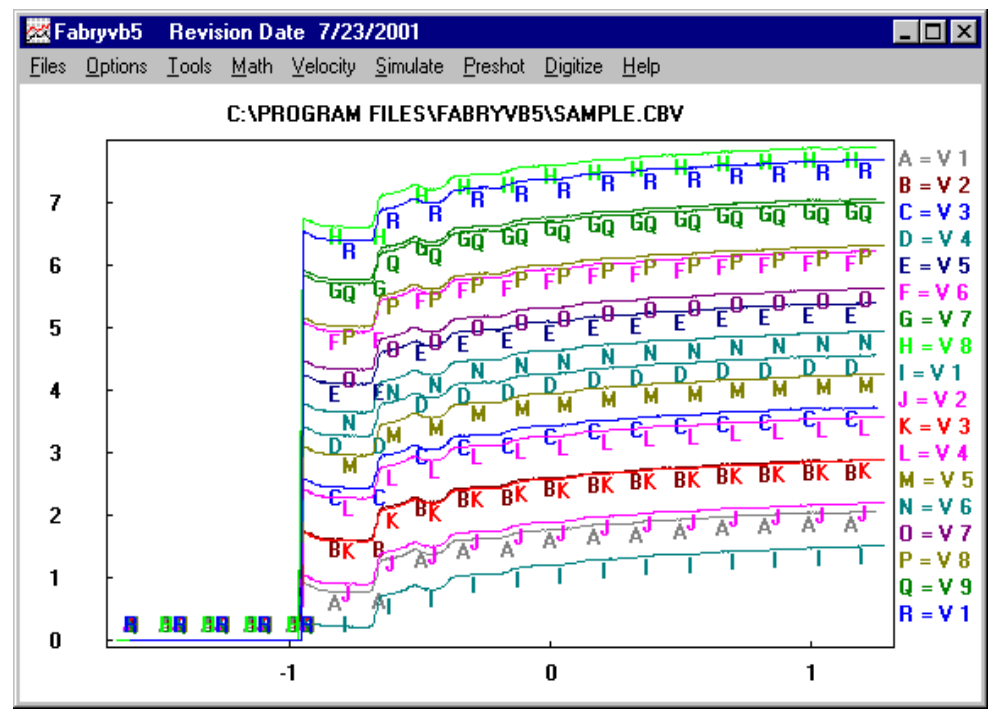

Figure 8. Overlay of sample.cav and sample.cbv Curve Sets 
The final velocity curve is plotted along with an error curve (see Fig. 9). The program multiplies estimated error values by 100 so that they can be plotted along with the velocity curve. Error values are calculated by applying partial derivatives to Equation 1

$$
F=\left(R^{2}-r_{1}^{2}\right) /\left(r_{2}^{2}-r_{1}^{2}\right) .
$$

Where $R$ is a Doppler shifted fringe (variable) and the $r$ 's represent UN-Doppler shifted fringes (constants). Summing up the partial derivatives and replacing each (which is just a constant multiplied by the variable $\delta$ ). Replacing each diameter $d$ in $\delta$ with its radius $r$ and setting this equal to a function $F$, we get Equation 2 .

$$
\Delta \mathrm{F}^{2} \approx\left[\Delta \mathrm{R}^{2} /\left(\mathrm{r}_{2}{ }^{2}-\mathrm{r}_{1}{ }^{2}\right)\right]^{2}+\left\{\left[\Delta \mathrm{r}_{1}{ }^{2}\left(\mathrm{R}^{2}-\mathrm{r}_{2}{ }^{2}\right)\right]^{2}+\left[\Delta \mathrm{r}_{2}{ }^{2}\left(\mathrm{r}_{1}{ }^{2}-\mathrm{R}^{2}\right)\right]^{2}\right\} /\left(\mathrm{r}_{2}{ }^{2}-\mathrm{r}_{1}{ }^{2}\right)^{4} .
$$

The program calculates an error $\Delta$ as being equal to one standard deviation from the average value. Since $R$ is a variable, its error is estimated as $\Delta\left(R_{2}{ }^{2}-R_{1}{ }^{2}\right)$ where $\left(R_{2}{ }^{2}\right.$ $-R_{1}{ }^{2}$ ) is a constant. The error curve $B$ in Fig. 9 is calculated by summing the variances of all fringes that are read to get a differential with an incremental error $(\Delta)$ yields an approximate to the variance of $F$ (see Equation 3).

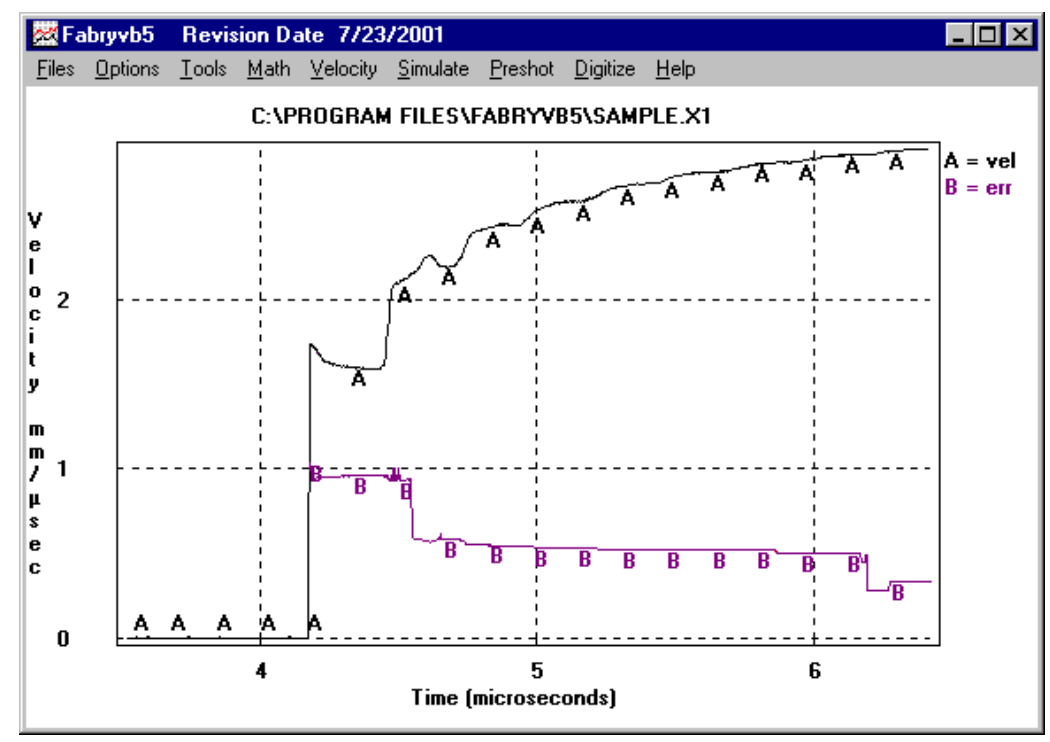

Figure 9. Velocity and Error Curves

A summary text file is created for the user to access more information on the quality of an analysis. Some important tabulation from the text file is shown in Table 1 and Table 2.

Table 1 lists the averaged radius-squared differences for UN-Doppler shifted fringes (curves 0a-1a) and for Doppler shifted fringes (curves $0 b-3 b$ ). Totsize is the number of data points in the curve and Rsqsize is the number of points calculated single velocity curve. Errors from a twofringe average will be approximately $2^{-1 / 2}$ times the errors for a single fringe. The downward steps in the error curve occur where the number of fringes (that are read) increase for a given time domain. 
their computed velocities should be exactly equal to an integer number of fringe constants. The error column tabulates these differences and it is an excellent overall check on the accuracy of velocity calculations. The largest errors will occur where the changing velocity jumps, causing a large difference in velocity for a small difference in time. The user can control sensitivity of crossover point calculations to the velocity slope by going to Tools / Curve Operations / Set Maximum Slope. Selecting Notepad from the File menu can access the text file discussed above.

\begin{tabular}{|llllll|}
\hline CURVE & AVERAGE & STD DEV & PERCENT & TOTSIZE & RSQSIZE \\
\hline C $=0 \mathrm{a}$ & 0.0556 & 0.0006 & 1.0 & 207 & 192 \\
$\mathrm{D}=$ 0a & 0.0566 & 0.0005 & 0.9 & 206 & 175 \\
$\mathrm{E}=1 \mathrm{a}$ & 0.0556 & 0.0006 & 1.1 & 191 & 191 \\
$\mathrm{~F}=1 \mathrm{a}$ & 0.0566 & 0.0005 & 1.0 & 175 & 175 \\
$\mathrm{G}=$ 0b & 0.0555 & 0.0004 & 0.7 & 171 & 156 \\
$\mathrm{H}=$ 0b & 0.0561 & 0.0005 & 0.9 & 163 & 145 \\
$\mathrm{I}=1 \mathrm{~b}$ & 0.0555 & 0.0007 & 1.2 & 587 & 156 \\
$\mathrm{~J}=1 \mathrm{~b}$ & 0.0561 & 0.0007 & 1.3 & 581 & 145 \\
$\mathrm{~K}=$ 2b & 0.0562 & 0.0026 & 2.7 & 666 & 525 \\
$\mathrm{~L}=$ 2b & 0.0570 & 0.0016 & 5.9 & 657 & 529 \\
$\mathrm{M}=$ 3b & 0.0564 & 0.0033 & 3.9 & 659 & 655 \\
$\mathrm{~N}=$ 3b & 0.0563 & 0.0022 & & 658 \\
\hline
\end{tabular}

Table 1. Radius Squared Differences

\begin{tabular}{|llcccc|}
\hline CUR-1 & CUR-2 & X & Y & VELOCITY & ERR(VEL) \\
\hline $\mathrm{C}=0 \mathrm{a}$ & $\mathrm{I}=1 \mathrm{~b}$ & .4738049 & .7317749 & 2.745767 & $3.110778 \mathrm{E}-03$ \\
$\mathrm{C}=0 \mathrm{a}$ & $\mathrm{I}=1 \mathrm{~b}$ & .4783973 & .7317749 & 2.753166 & $4.288502 \mathrm{E}-03$ \\
$\mathrm{C}=0 \mathrm{a}$ & $\mathrm{I}=1 \mathrm{~b}$ & .5449615 & .7317749 & 2.759646 & $1.076795 \mathrm{E}-02$ \\
$\mathrm{C}=0 \mathrm{a}$ & $\mathrm{I}=1 \mathrm{~b}$ & .5620036 & .7317749 & 2.764953 & $1.607492 \mathrm{E}-02$ \\
$\mathrm{D}=0 \mathrm{a}$ & $\mathrm{J}=1 \mathrm{~b}$ & .4487458 & .2684139 & 2.747039 & $1.838351 \mathrm{E}-03$ \\
$\mathrm{D}=0 \mathrm{a}$ & $\mathrm{L}=2 \mathrm{~b}$ & -.6470613 & .2684139 & 2.076888 & $1.522964 \mathrm{E}-02$ \\
$\mathrm{E}=1 \mathrm{a}$ & $\mathrm{K}=2 \mathrm{~b}$ & .3891999 & .8306894 & 2.733716 & $1.516213 \mathrm{E}-02$ \\
$\mathrm{E}=1 \mathrm{a}$ & $\mathrm{K}=2 \mathrm{~b}$ & .4408722 & .8306894 & 2.74801 & $8.678916 \mathrm{E}-04$ \\
$\mathrm{~F}=1 \mathrm{a}$ & $\mathrm{L}=2 \mathrm{~b}$ & .435573 & .1680038 & 2.748459 & $4.187901 \mathrm{E}-04$ \\
$\mathrm{~F}=1 \mathrm{a}$ & $\mathrm{L}=2 \mathrm{~b}$ & .5534215 & .1680038 & 2.757058 & $8.180824 \mathrm{E}-03$ \\
\hline
\end{tabular}

Table 2. Crossover Point Statistics

\section{Back Calculating Fringes from a Velocity Curve}

Fringes can be back calculated from a velocity curve using routines in the Simulate menu. This is very useful for checking the correctness of cavity sorting. The calculated fringes should exclusively overlay onto the original bitmap image. If there are extra calculated fringes or if there is a shortage of calculated fringes, then an error has occurred in the cavity sorting.

For back calculation, we must extract the fractional orders from the original fringe 
curves. First, read up the file sample.cb and select Tools / Evaluations / Fractional Order. Next, do the same for the sample.ca curve. We have now loaded up values of fractional orders for the primary and referee cavity fringes respectively. Now, read up the sample.udv curve and select Simulate /
Settings from the menu. Figure 10 shows the dialog for input on simulations. The user must type in values for the two cavity lengths, the fid period and optical parameters. Clicking OK will calculate the fringe constants. Clicking Simulate / Make Fringes will calculate the fringes.

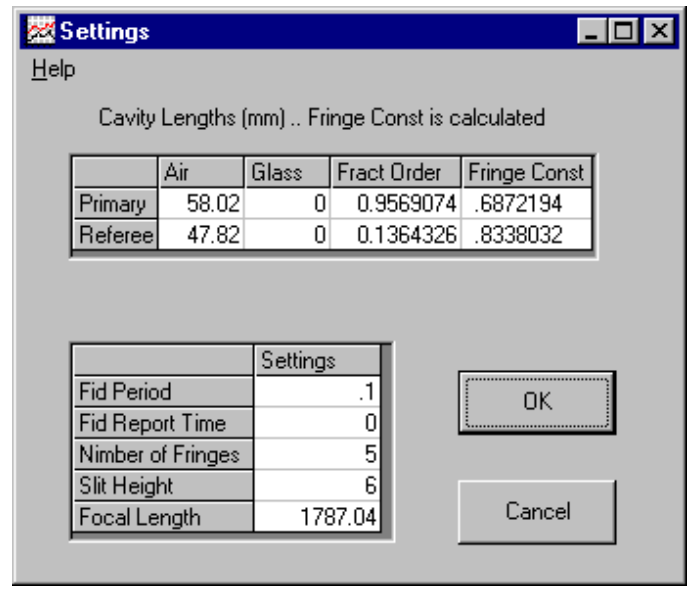

Figure 10. Dialog for Input on Simulations

The program overlays the two calculated fringe files samplesim1.sim and samplesim2.sim. These files are for the primary and referee cavities respectively.

Finally, read up the original bitmap file and select File / Overlay to overlay either (or both) of the simulated fringe files. Figure 11 shows the overlaid fringes after adjustments have been made on magnification and translation. To translate a fringe file, select
Simulate / Move $X, Y$ Overlay from the menu and then click and drag with the mouse. Similar operations will perform Stretch $X$ and Stretch $Y$ adjustments. A small circle near the center will indicate where to click for a new translation move. Small differences between the overlays and the original image occur where distortions are in the bitmap image. The main purpose is to account for all fringes in the calculation.

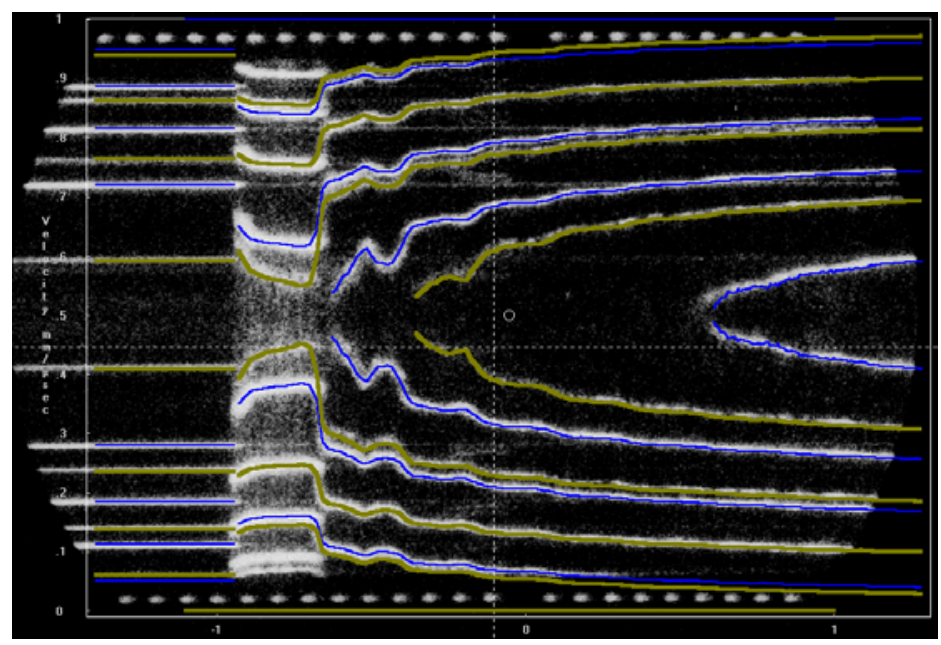

Figure 11. Fringe Simulation Overlay 


\section{Many Beam Velocimeter Records}

All of the routines discussed so far also apply to analyzing records produced by the Many Beam Velocimeter. The only difference between a Many Beam analysis and a Single Record analysis is that the user will select Many Beam Auto Analysis instead of Single Record Auto Analysis from the Velocity menu. The same dialog will appear as before and the user must fill in all applicable fields. Figure 12 shows the input dialog that is completed for a different 10 beam record analysis.

Note that the table-2 cavity length data includes a 20.82-mm thick glass insert, which causes one side of the primary cavity to become a referee cavity of increased optical thickness. The air thickness for the referee cavity is just the air thickness of the primary cavity $(58.06-\mathrm{mm})$ less the glass thickness.

Velocities are still calculated from Equation (1). The cavity thickness $h$ is automatically replaced with $h+T(n-\lambda d n / d \lambda)$, where $n$ is the index of refraction for a glass insert of thickness $T$ (see Ref. 4).

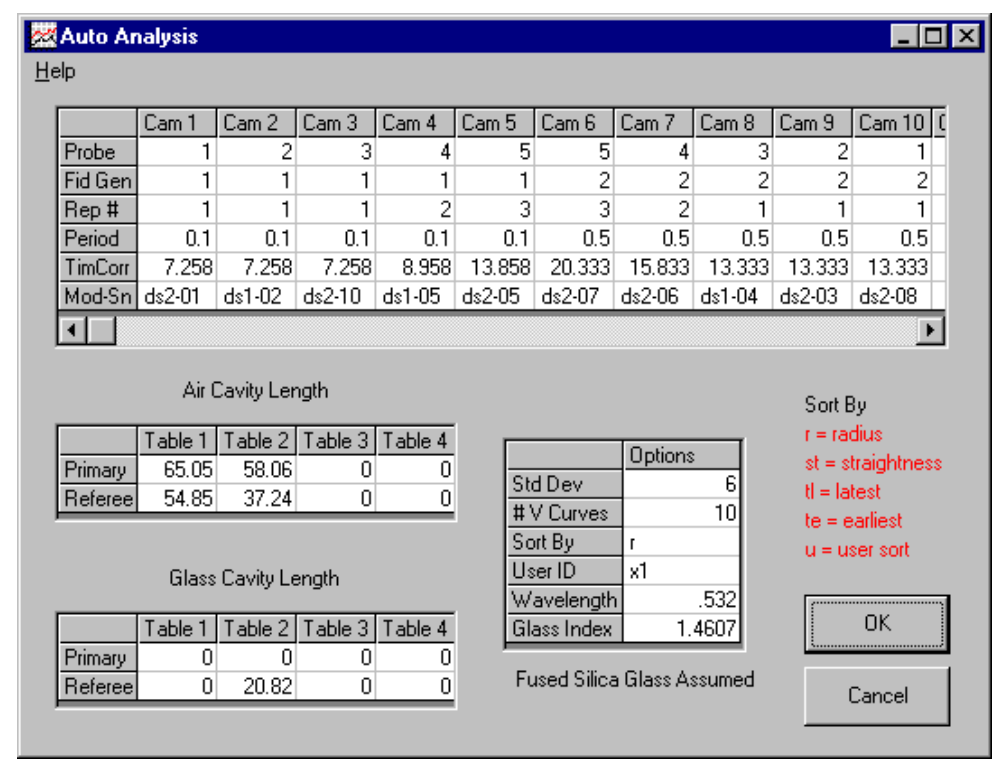

Figure 12. Editing sample.dat for Many Beam Auto Analysis

More beams can be added up to a total of 20 cameras. When more than 20 beams are needed, double records can be utilized for each camera to increase the total number of beams to 40 on a four table Volicimeter. Multiplexing two beams onto one camera is accomplished by an optical technique that projects one beam onto the upper half and another beam on the lower half of single slit.

The analysis program recognizes double record data when the user selects Double Records On Camera from the Velocity / Auto Analysis Options menu. Examples and instructions on file naming conventions for Many Beam Records and Double Records are found in the Help menu on Record Analysis and on Double Records.

After completing the analysis of all 10 records, the user can select Velocity / Make Shot Analysis Summary to summarize all data for the record set. Data are extracted from each of the camera text files and are tabulated into summary tables.

Selecting Velocity / Batch Auto Analysis allows the user to re-calculate the velocity on all fringe curves. This is useful when a new parameter (such as interferometer cavity length or laser wavelength) is to be used to re-calculate the original data. 
Distortion correction curves can be applied during an analysis by pre-selecting Apply Un-Distort $X$ Curves and/or Apply Un-distort $Y$ Curves from the Velocity / Auto Analysis Options menu. The $X$-axis is time and the $Y$ axis is space on the fringe file sample.ult.

Distortion correction curves that are currently available are approximately one year old and they are expected to degrade over time (see Reference 3). One can evaluate their current effectiveness by comparing velocity errors from the crossover point statistics. If the errors are larger for velocities calculated using distortion correction curves then the curves should not be used and new calibrations are needed. Appendix A describes the routines and methods used to create new distortion correction curves.

When using distortion correction curves, it is important to verify that the scanner to be used in obtaining a bitmap image is sufficiently distortion free for the analysis. The film must also be scanned from the emulsion side of the film record, otherwise the top and bottom of the image will be reversed and distortion corrections will be invalid.

\section{Using the Preshot Menu}

The Preshot menu was created to calculate streak camera set up timing and to create the sample.dat file. Users can open LabView or Catalyst digitizer data files to measure timing information. Reading a Catalyst file requires that the executable file cattext.exe must reside in the folder containing the Catalyst file being read.

Using the input dialog's default setting for minimum volt amplitude will work for most LabView or Catalyst files. Entering a zero will cause the default setting to be applied for the analysis. Select Analyze Times to determine times between the first signal and all later signals. The program's sensitivity to interpreting noise as a signal can be reduced by selecting Reset Signal Level from the Preshot menu. Again, entering a zero will cause the default setting to be applied.

Selecting Create / Modify Setup File from the Preshot menu loads up the input dialog shown in Fig. 13.

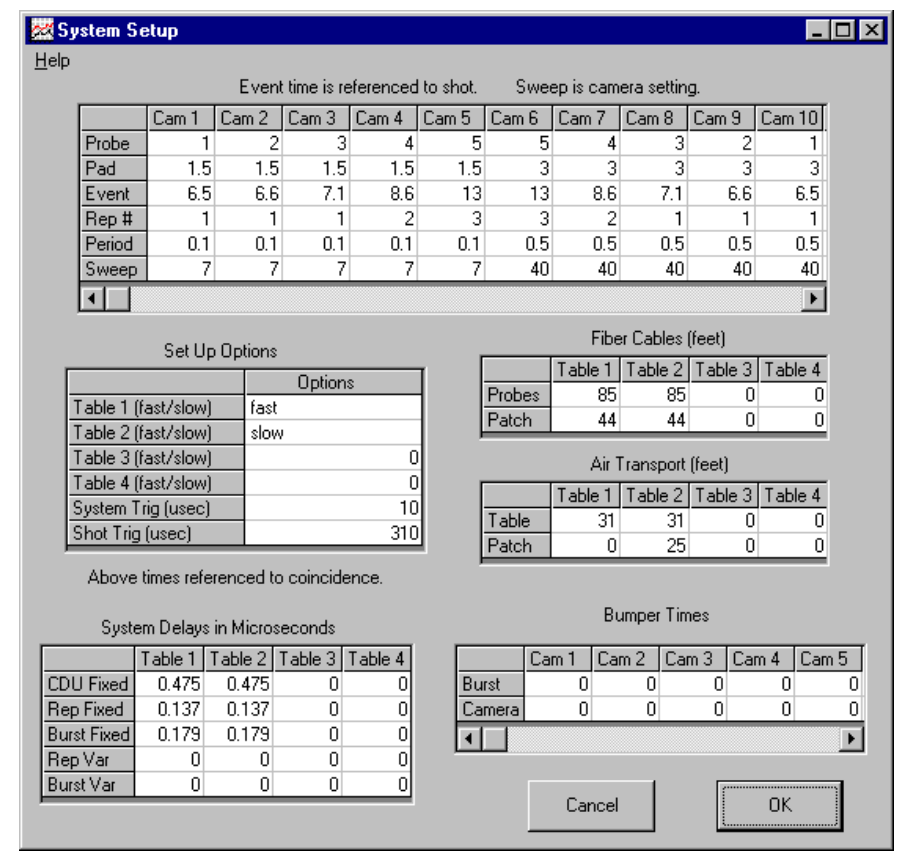

Figure 13. Editing the sample.set File 
This dialog allows us to create or modify the set up file sample.set. All input times are in microseconds. Pad (in the first block of Fig. 13 ) is the time from the first fid dot to the Event. Period is the time between fid dots and Report (the time reference) is the number of dots that are dimmed on the fid burst (string of dots).

The Setup Options block of Fig. 13 allows selection of slow or fast for the table set up timing. The fast set up is required when the camera start times must float with the timing jitter of a given fire-set. This option requires that the time between the shot CDU and the streak camera start time must be greater than $1 / 10$ of the streak camera sweep setting. This is necessary because a streak camera trigger time starts the camera streak tube rather than the intensifier tube (which is smaller in diameter). Using the slow option only requires that the operator set up enough Pad time to compensate for fire-set time jitter. System Trig is the timing set up signal sent from the control room and must be at least 300 microseconds earlier than the earliest Event time. This allows time for the pulsed laser to be producing useable light. Shot Trig is the time that the shot CDU fires the shot.

The System Delays box must have values typed in for signal time delays of the shot CDU, the fid Report and the fid Burst. Report Var and Burst Var are user input values of the variable delays (signal cables) that are installed into the system signal cables to zero out (correct) time delays for a given shot. Other boxes allow input of fiber optic cable lengths and laser air-transport lengths. Delay times are calculated for these values. Bumper Times are for trimming delay times during dry times runs, i.e. correcting for individual function times of the fid bursts and streak cameras.

Clicking $O K$ will update/create the sample.set and sample.prn files. A text editor automatically opens the sample.prn file onto the monitor screen. This file summarizes all of the set up information.
Selecting Create Dat File from the Preshot menu causes the program to read data from the sample.prn file as input for creating the sample.dat file. Once the program opens the input dialog (see Fig. 12) the user must type in the raw measured times between the shot CDU and the report for each camera (the TimCorr row). The streak camera model/serial numbers should also be typed in. Interferometer cavity lengths for each table that is used must be typed into the appropriate blocks. Clicking $O K$ will generate the sample.dat file with the appropriate corrections made for system time delays.

Tables 3-5 show the contents of our sample.prn file discussed above. Table-3 lists interferometer data and timing corrections on fid report times. Table-4 lists calculated delay times and settings for the streak cameras. Table- 5 lists signal cable time corrections along with input from the sample.set file. The sample.prn file and the sample.dat file should be forwarded to the analyst along with the streak camera shot records.

We close with a word on using the mathematical functions and other routines to step through an analysis. Math functions are included in the program to support the analysis routines. Fig. 14 shows the various math functions that can be selected from the menu. Most menu selections begin with an input dialog that explains what it will do and then prompts for whatever input that it needs.

The user can step through a complete analysis by selecting various routines, one at a time. Additionally, any single function (math of otherwise) can be used to perform an operation on the currently opened file. An input prompt will explain what type of file that the function can work on or will open an input dialog with a help menu. The user will then have a choice to continue or to exit the function without doing anything. The Help on Record Analysis contains step by step instructions for doing this type of analysis. 


\begin{tabular}{|c|c|}
\hline Math Velocity Simulate Preshot & Digitize $\underline{H}$ elp \\
\hline Curve Operations & \multirow{4}{*}{$\begin{array}{l}\text { Append Curves ... } \\
\text { Sum of Curves ... } \\
\text { Sum One Curve to All Others ... } \\
\text { Difference of Two Curves ... }\end{array}$} \\
\hline Multinlu X hu const & \\
\hline Multiply Y hu const... & \\
\hline & \\
\hline \multirow{3}{*}{$\begin{array}{l}\text { Add const to } \underline{X} \ldots \\
\text { Add const to } \underline{Y} \ldots \\
\text { Add Fringe Constant } \underline{\text { to } Y \ldots}\end{array}$} & \multirow{2}{*}{$\begin{array}{l}\text { Product of Two Curves... } \\
\text { Quotent of Two Curves... }\end{array}$} \\
\hline & \\
\hline & \multirow{3}{*}{$\begin{array}{l}\text { Square of Curves... } \\
\text { Square Root of Curves ... }\end{array}$} \\
\hline Absolute Value $X \ldots$ & \\
\hline Absolute Value $Y . .$. & \\
\hline Normalize X Values to 1 & \multirow{3}{*}{$\begin{array}{l}\text { Integrate Curves ... } \\
\text { Differentiate Curves ... } \\
\text { Cubic Spline Smooth ... }\end{array}$} \\
\hline Normalize $Y$ Values to 1 & \\
\hline Normalize All Values to 1 & \\
\hline Normalize $\times$ Values to Entry & \multirow{3}{*}{$\begin{array}{l}\text { Best Polynomial Fit ... } \\
\text { Specific Polynomial Fit ... } \\
\text { Include Max/Min on Plots } \\
\text { Extend Curve from Fit ... }\end{array}$} \\
\hline Normalize Y Values to Entry & \\
\hline \multirow[t]{2}{*}{ Swwap $X / Y$ Axes } & \\
\hline & Intercept of Two Curves. \\
\hline
\end{tabular}

Figure 14. The Math Menu

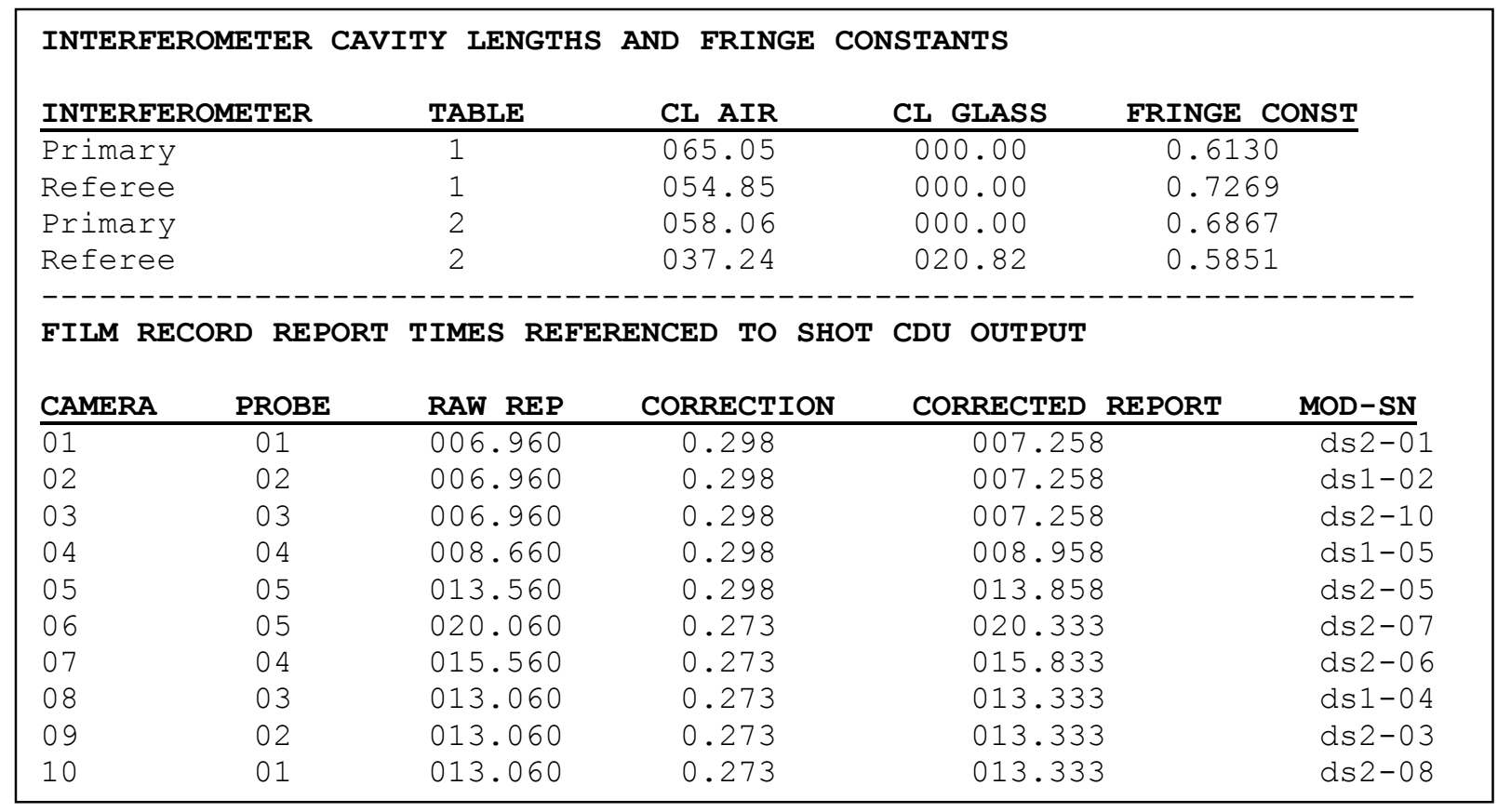

Table 3. Part of the sample.prn File 


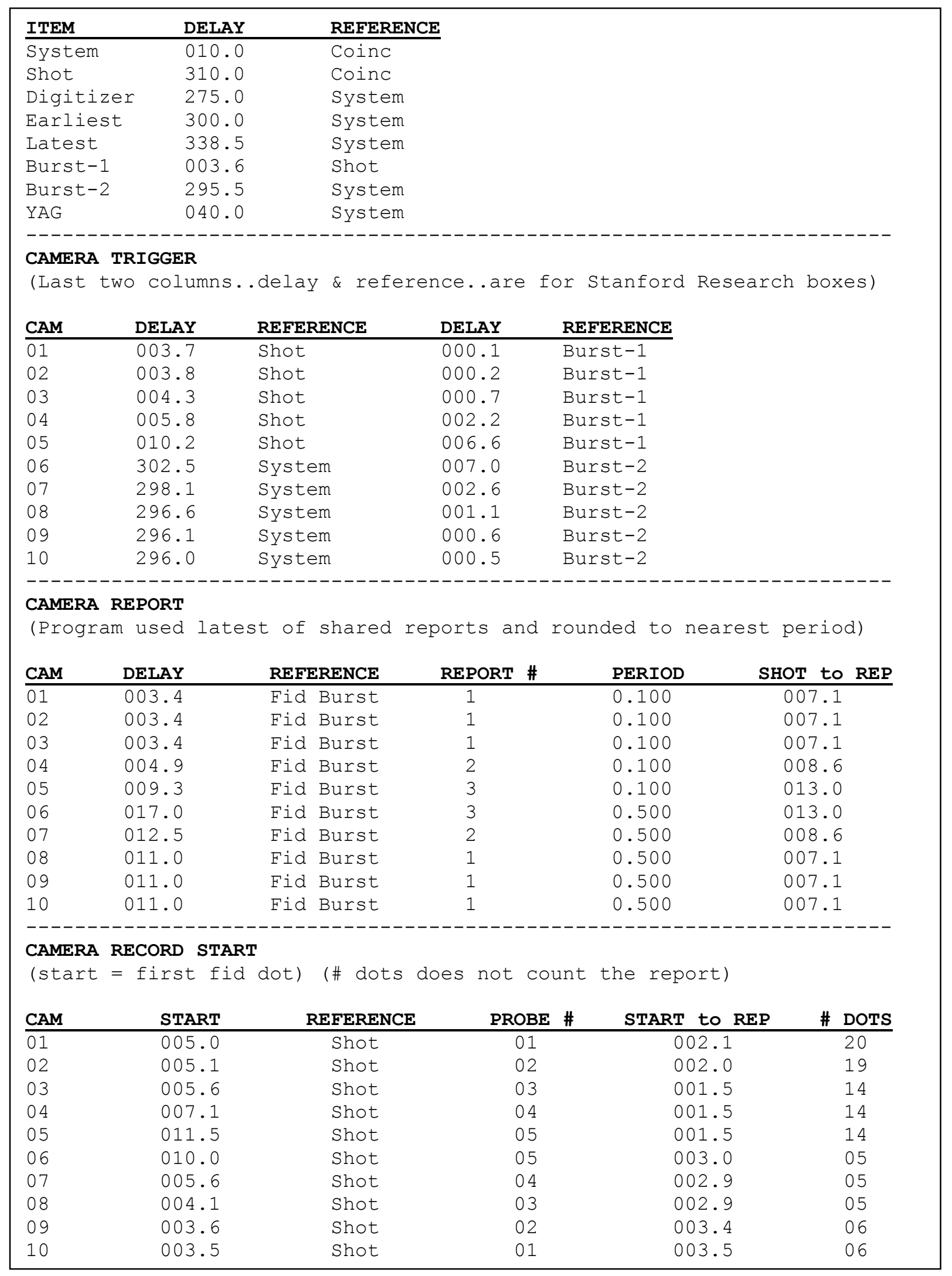

Table 4. Part of the sample.prn File 


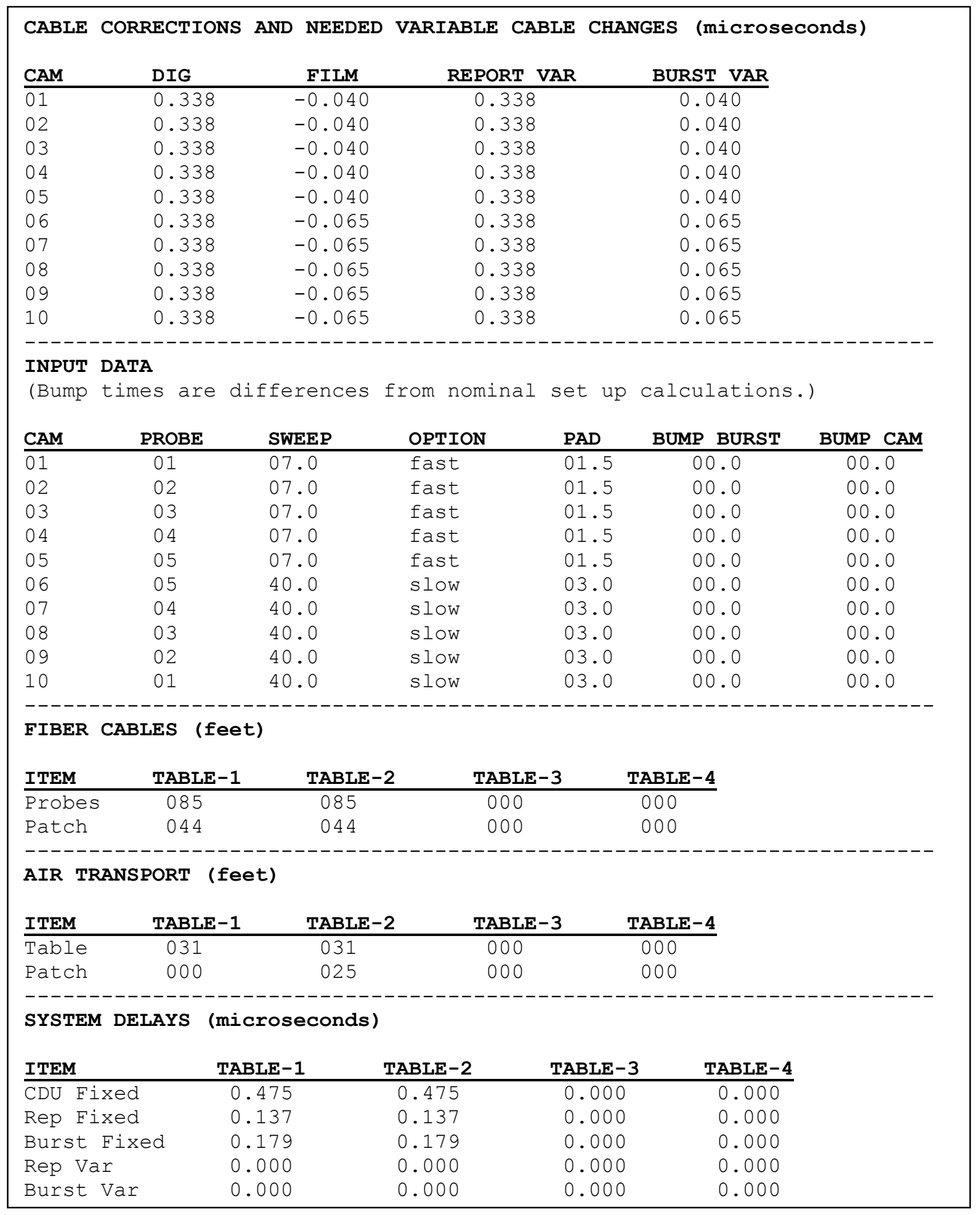

Table 5. Part of the sample.prn File 


\section{Appendix A: Streak Camera Distortion Calibrations}

Results of streak camera distortion calibrations can be found in Reference 3 . Here, we will describe program routines used to analyze distortion calibration records and to create distortion correction curves.
Fig. A1 shows the reading of a time distortion calibration record where we simply read from top to bottom using the Fids selection of the Digitize menu.

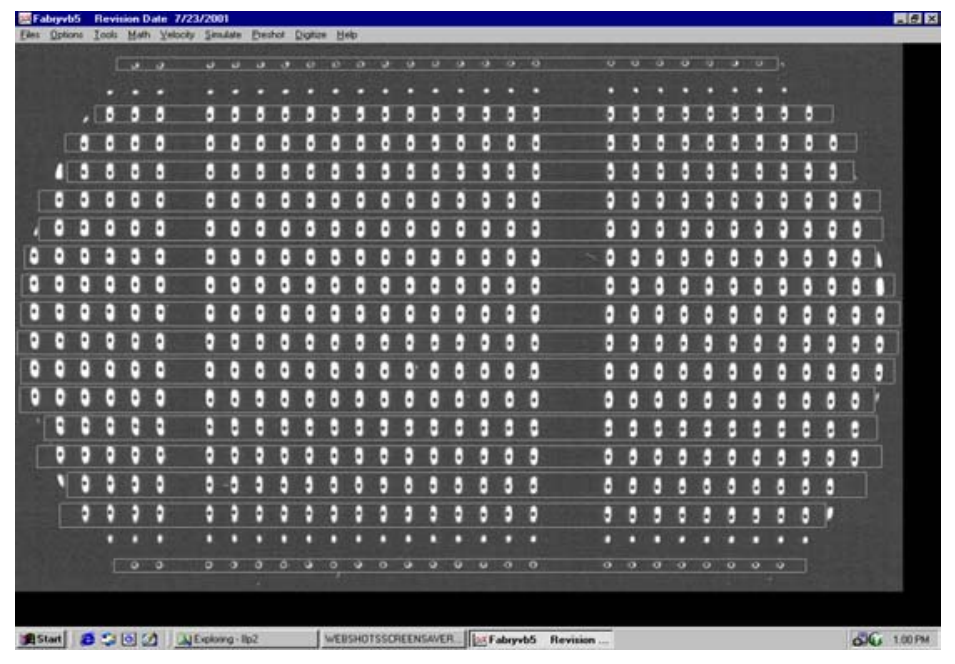

Figure A1. Time Distortion Calibration Record

After quitting the Digitize menu, select Make Undistort X Curve Set from the Tools / Fid Operations menu. The program calculates a time distortion correction file * $x d s$ where ${ }^{*}$ is the applicable name of the bitmap file. Contents of the *.$x d s$ file are described in Reference 3. Several such files can be averaged by selecting Tools / Average Many Un-Distort Curve Sets. Next, we test the averaged correction curve set by reading up the ${ }^{*}$.ult file that was created from the original bitmap image. Selecting Tools / Rename Curves Like Fringe File will prepare the file for testing.

Select Options / Un-distort / Do All Above to perform the standard correction using only the top and bottom fids. Follow this correction by selecting Apply Un-Distort $X$ Curve Set from the Tools / Fid Operations menu. The resulting corrected curve can be evaluated by selecting Deviation From Nominal X Many Fids from the Tools / Fid Operations menu. The resulting curve is a plot of time errors $(Y)$ for the time axis $(X)$. If there are wild points at the ends of the curves (due to edge distortions of the intensifier) we can remove them by selecting Max Distortion Or Error from the Tools menu.

Similarly, we create a set of space (Y-axis) correction curves from a space distortion calibration record (see Fig. A2). On this record, we must use the Auto Curve Fit menu selection to read the lines. Top and bottom fids are read as usual.

To make the space correction curves, select Make Un-distort Y Curve Set from the Tools IY Axis Operations menu. To test an averaged set of space correction curves, read up the *.ult file and select Tools / Rename Curves Like Fringe File. Now select Options / Un-distort / Do All Above followed by Apply Un-Distort Y Curve Set from the Tools / Y Axis Operations menu.

Evaluate the corrected curve by selecting Deviation From Nominal $Y$ for Lines from the Tools / Y Axis Operations menu. The resulting curve is a plot of space errors $(Y)$ for the time axis $(X)$. Make a qualitative evaluation by reading up the corrected (and 
saved) file followed by selecting Overlay Nominal $X$ or $Y$ Lines from the Tools menu. This can also be done for a time corrected curve set. Graphical plots of the qualitative overlay can be found in Reference 3. Note that any correction curve set ( ${ }^{*} . x d s$ or $\left.{ }^{*} . y d s\right)$ can be scaled to a different time domain by selecting Scale Distortion Curves from the Velocity menu.

We check the effectiveness of our space correction curves using velocity calibration records such as that shown in Fig. A3. These types of records are also discussed in Reference 3. We read the calibration record using the Auto Curve Fit selection from the Digitize menu. Weak lines represent Doppler shifted fringes and bright lines represent UN-Doppler shifted fringes. For the analysis, one must read at least two pair (one pair is a top and bottom fringe set) of UN-Doppler lines and three or four pair of Doppler lines. Read from the middle regions out towards the fids. Outer regions near the fids are usually too weak and crowded for a good analysis. Readings can be in any order but the fids should be read first.

To analyze the resulting *.ult file, select Double Records on Camera and Apply UnDistort Y Curves from the Velocity / Auto Analysis Options menu. Now select Analyze Velocity Calib Curves from the Tools / Y Axis Operations menu.

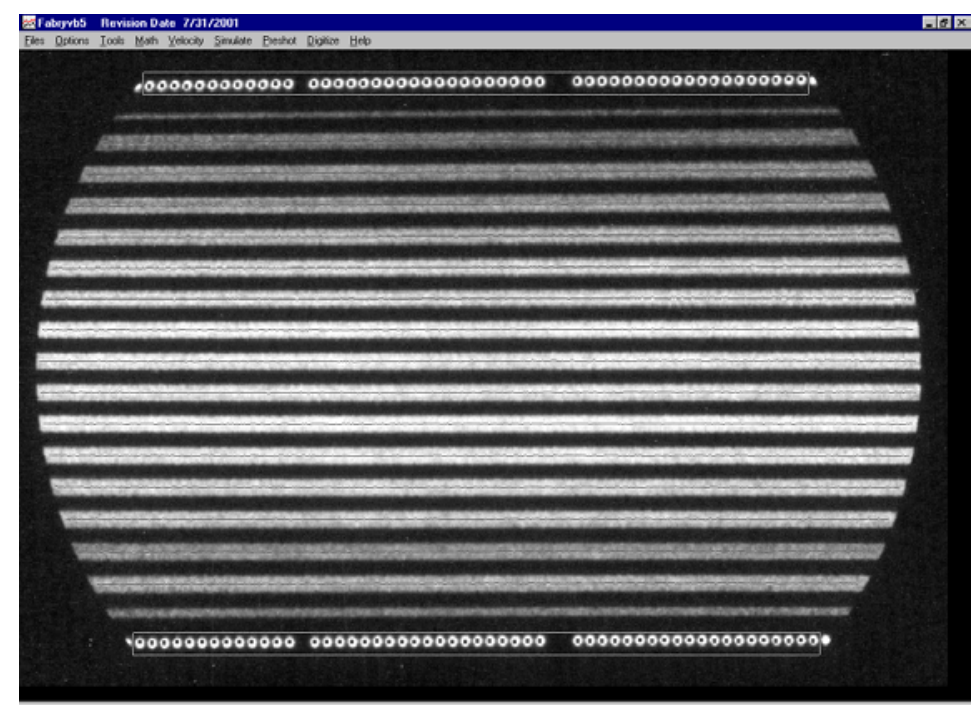

Figure A2. Space Distortion Calibration Record

A series of operations will occur while the screen displays some calculated curves. When the analysis stops, read up the resulting *.txt file using the File / Notepad menu. Discussion and interpretation of this file can be found in Reference 3.

The calibration record shown in Fig. A3 was read with three pair of UN-Doppler lines. Using three pair of UN-Doppler lines, the program can calculate the center of the fringe pattern by using only the top half or the bottom half of a record (see Fig. A3).
Equation A1 solves for $r$ by using only the parameters $a$ and $b$.

When there are only two pair of UN-Doppler lines used, the user must select Use Auxiliary Record for Centering from the Tools menu before doing the analysis. An auxiliary record is a streak camera photographic exposure of a full fringe pattern (not the top half of one fringe set plus the bottom half of another fringe set). A value for the precise vertical center position of a fringe pattern is required for analysis of any double record.

$$
\text { (A1) } r=a b /(a-b)-1 / 2(a+b) \text {. }
$$


We end with a word on correcting distortions by using three fids (top, middle and bottom) instead of the full map of fids as described in Reference 3. Distortion correction files can be made from a standard velocity record provided that the three fids have been installed onto the streak camera slit plate.
By selecting Make 3 Fid Distortion Curves from the Velocity menu, we can create a set of distortion correction curves from the three sets of fid dots. Reference 3 summarizes the effectiveness of this method of distortion correction.

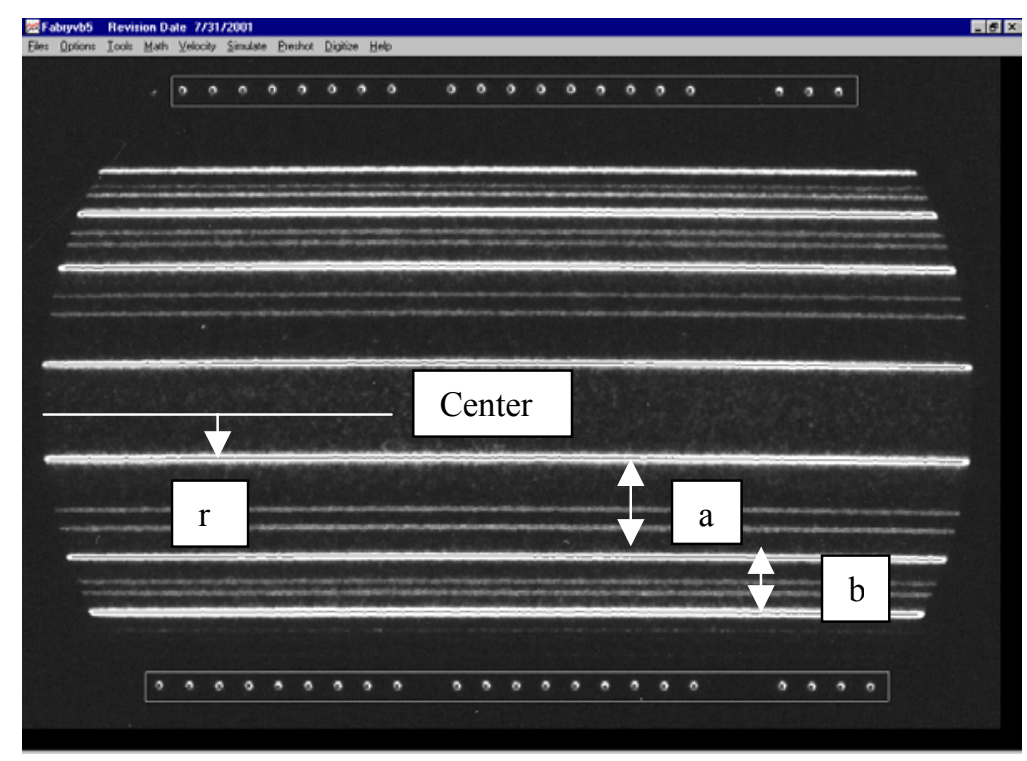

Figure A3. Velocity Distortion Calibration Record

\section{References}

1. C. F. McMillan, D. R. Goosman, N. L. Parker, L. L. Steinmetz, H. H. Chau, T. Huen, R. K. Whipkey, and S. J. Perry, Rev. Sci. Instrum. 59, 1 (1988).

2. David Goosman, George Avara, Lloyd Steinmetz, Ching Lai, Stephen Perry, "Manybeam Velocimeter for Fast Surfaces", $22^{\text {nd }}$ International Congress on High-Speed Photography and Photonics, SPIE 2869, 1070 (1996).

3. George Avara, Leland Collins, Anthony Rivera, "Distortion Corrections for the Many Beam Fabry Perot Velocimeter", UCRL-ID-143225 (March 21, 2001).

4. David Goosman, Lloyd Steinmetz, Stephen Perry, "Striped Double Cavity Fabry-Perot Interferometers Using Both Glass and Air", $23^{\text {nd }}$ International Congress on High-Speed Photography and Photonics, SPIE 3516, 296 (1998). 